\title{
تحليل صعوبة في تعلم مهارة الكلام عند تخصص اللغة العربية في معهد دار السلام
}

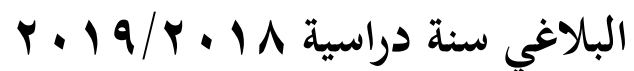

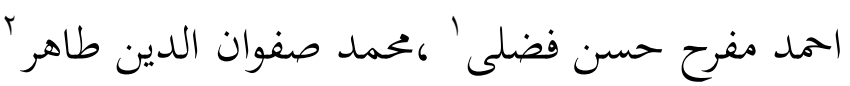 \\ جامعة الإسلامية دار السارم البلاغي
}

Sofanudintohir.96@gmail.com, ahmadmufarih@iaida.ac.id

$$
\begin{aligned}
& \text { مستخلص البحث } \\
& \text { كان الغرض من هذا البحث هو معرفة مسائل أو صعوبات التي تواجه المشترك } \\
& \text { اللورة في دورة اللغة العربية دار السلام (كارد) و طريقة حلها. نوع البحث المستخلدم } \\
& \text { هو البحث النوعي الني أجري باستخدام ثلاث تتنيات لجمع البيانات، وهي المقابلات } \\
& \text { والملاحظة والوثائق. وطريقة تحليل البيانات المستخلدمة هي النوعي الوصغي. بناءً على } \\
& \text { نتائج الملاحظات والمقابلات التي أجريت في دورة اللغة العربية دار السلام (كارد)، فإن }
\end{aligned}
$$

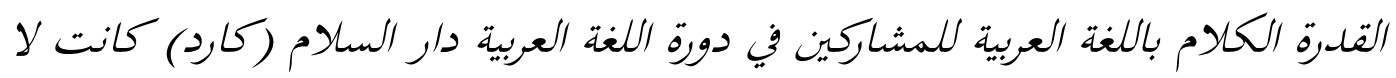

$$
\begin{aligned}
& \text { تنال تعنبر مغقودة بسبب علة عوامل وهي قلة حغض المفردات العربية وقلة فهم القواعل } \\
& \text { اللغوية وعلدم اهتمام الطالب بتعلم اللغة العربية، وما إلى ذلك. . وطريقة حل صعوبات } \\
& \text { لتعلم مهارة الكلام لمشتركين اللدوة اللغة العربية دار السلام (كارد) وهي بإكثار حغظ } \\
& \text { المفردات و إكثار النطبيق اللغوبة واشتراك الندوات، المناقشات والدراسة المخصصة. } \\
& \text { الكلمات المفتاحية: تحليل صعوبات، مهارة الكالام، دورة اللغة العربية. }
\end{aligned}
$$

Abstrak

Tujuan dari penelitian ini adalah untuk mengetahui apa saja problem atau kesulitan yang dialami dalam mempelajari bahasa Arab di Kursus bahasa Generasi Arab Darussalam (GARD). Dan cara mengatasi kesulitan tersebut.Adapun jenis penelitian yang digunakan adalah penelitian kualitatif deskriptif yang dilakukan dengan tiga teknik pengumpulan data yaitu wawancara, observasi dan dokumentasi. Dan hasil penelitianya yaitu Kemampuan berbicara bahasa Arab di Kursus bahasa Generasi Arab Darussalam (GARD) dapat meningkat jika peserta kursus menyadari akan kebutuhan berbahasa Arab.

Kata Kunci: Kesulitan, Kemahiran Berbicara, GARD. Berbahasa Arab. 
مهارة الكلام هي إحدى المهارات اللغوية وهي نوع من المهارة على تحقيقه في تدريس اللغة. الكلام هو الوسيلة الرئيسية لتعزيز التفاهم المتبادل والتواصل الإجتماعية كذلك بواسلة اللغة. تشتمل أنشطة الكلام على جوانب من الاتصالات ثنائية الاتحاه، وهي الكلام أو التحدث مع المتكلمين الأخرى. (أفندي و الأخر : با • ץ: 107). وبالتالي يجب أن تستند تمارين الكلام أولاً إلى المهارة الاستماع يواصل على مهارة الكلام، وإتقان المفردات والعبارات التي تسمح للمشاركين في الدورة تعبيرا عن نواياهم وأرائهم وأفكارهم. لذلك نقول أن ترين الكلام هو استمرار لممارسة الاستماع التي تتضمن أيضًا تمارين مهارة الكلام و التحدث.

نظرا إلى اللغة العربية هي اللغة أنبل وأكثر المفردات بالنسبة إلى لغة أخرى، حتى عملية وقائمة دورات هذه اللغة يتطلب إلى أساليب التعلم المناسبة والفعالية. أما أمر التعلم هي إيصال المعرفة باللغة العربية بين المعلم والمتعلم في كثير من الأحيان يواجه المتعلم صعوبات أو مشاكل اللغوي. كمثال على صعوبات الكلام التي هي مور الباحث، فإن هذه الصعوبات ناتجة عن عوامل داخلية للمشاركين في الدورة أو المشاركين الخارجي ينفي الدورة نفسها. المسئلة أيضا يأتي من عامل اللغوي العربية نفسها التي تشمل نظام الصوت، اللغوي، والكتابة 
والمفردات و أيضا معامل عدم اللغوية التي تشمل التعليمية والاجتماعية والثقافية

$$
\text { والسياسية والنفسية (أقندي والأخر r | • ب : بד (). }
$$

إن ادعاءات الباحث بأن المشاركين في الدورة اللغة العربية (كارد) واجهوا

صعوبات في اتقان مهارة الكلام تكون لها تأثير لاحق على رغبة أو تحفيز المشاركين في الدورة اللغة نفسها. يهتم الباحث ئ الدراسة بدراسة الصعوبات في تعلم مهارات الكالام من حيث اللغوي و غير اللغوي عند دورة اللغة العربية دار السالام (كارد).

بناءً على نتائج المالاحظات والمقابلات التي أجريت في دورة اللغة العربية دار السلا (كارد)، فإن القدرة الكاملام باللغة العربية للمشاركين في دورة اللغة العبية دار السالام (كارد) كانت لا تزال تعتبر مفقودة بسبب عدة عوامل وهي قلة حفض المفردات العربية وقلة فهم القواعد اللغوية وعدم اهتمام الطالب بتعلم اللغة العربية وما إلى 
ا ـما هي الصعوبات التي تعترض تعلم مهارة الكلام في دورات اللغة العربية بمعهد

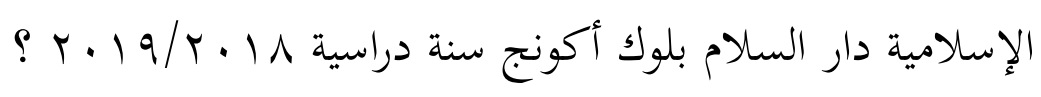

r. ما هي خطوات لحل الصعوبات التي تعترض تعلم مهارة الكالام في دورات اللغة

$$
\text { العربية معهد الإسلامية دار السلام بلوك أكونج سنة دراسية م | • / و | • ب ؟ }
$$

ج.فوائد البحث

$$
\text { تنقسم فوائد هذه الدراسة إلى جانبين: }
$$

ا. نظريا يعنى من خلال هذا البحث، يمكن أن نتوقع تحسين وتعزيز

الاهتمام بالمشاركين و الكتاب في الدورة تعلم اللغة العربية بعد معرفة المشاكل

$$
\text { وكيفية الحل عليها. }
$$

r. عمليا يمكن أن يكون هذا البحث عبارة عن بيانات للدورة في معهد دار السلام

في الجهود المبذولة لتطوير اللغة ويمكن أن تستخدم أيضًا كمواد مرجعية وأساس للمعلمين والمشاركين في الدورة حسب معرفة العلمية ومصادر المعلومات.

ب. للكاتب أو الباحث عسي أن تكون هذه الأطروحة و هذا البحث علم ومعرفة جديدة للباحث و أن يكون خبرة طيبة لنمو كفائة ومقدرة كتابة الباجث

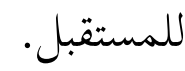


أطروحة كتبها نون إلهام شعبان تحت العنوان تحليل العوامل المثبطة للمهارة على

الكالام باللغة العربية للفصل الحادي عشر ملدرسة العالية الإملامية البلدية، لومبوك

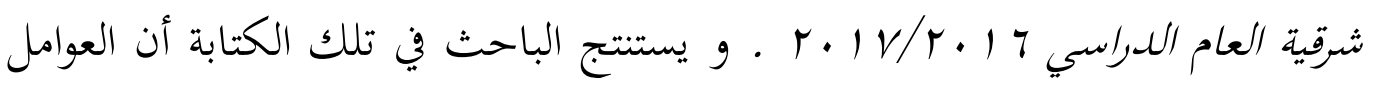

التي تسبب صعوبات لغوية هي: أقل إتقان المفردات، عدم التفاعل يستخدم اللغة العربية، بيئة اللغة،قلة ساعات الدرس، عدم الاهتمام بتعلم المشاركين في الدورة.

الأطروحة التي كتبها سيف الدين تحت العنوان تعلم مهارات الكالام المنهجي في

بحال دراسات اللغة العربية في معهد الفيفا لتعليم تكنولوجيا المعلومات كوا ع ا • ب . يستنتج الباحث أن العوامل التي تسبب صعوبات لغوية هي لا تزال قائمة على الصعوبات، اللغوية وغير اللغوية، عدم التعود في المحادثة اليومية باستخدام اللغة العربية. هـ إطار النظري |

التحليل في أي شكل من أشكال نوع الدراسة التي يتم إجراؤها هو طريقة لوجهة نظر أو تفكير، يتعلق بالتفكير المنهجي لشيء ما لتحديد العلاقة بين جزء واحد مع جزء آخر. Y ب الصعوبة

صعوبات التعلم هي حالة بتعل الطالاب لا يتعلمون كما ينبغي (أبو

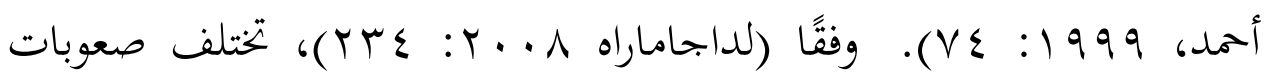


التعلم التي يشعر بها الطلاب، والتي يمكن تصنيفها إلى أربعة أنواع، وهي نظرا إلى نوع الصعوبات التعليمية، توجد صعوبات شديدة ومعتدلة،انطلاقا من الموضوعات التي تمت دراستها، هناك دائمة وبعضها مؤقت، انطلاقا من طبيعة الصعوبات، فهناك تلك التي تعيش في الطبيعة بشكل مؤقت، إذا نظرنا إليها من الأسباب، فبعضها يرجع إلى الذكاء والبعض الآخر عن عوامل غيره.

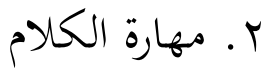

مهارة الكلام يشار أيضًا باسم التابير غالبًا. ومع ذلك، كل منهما تأكيدات مختلفة، حيث يشدد الكلام على القدرة اللفظية، في حين يمكن التعبير جانب اللفظي في شكل مكتوب ب. - م. الغرض من تعلم مهارة الكلام

تعلم التحدث بالعربية له عدة أهداف منها من أجل أن تكون قادرة على نطق التعبيرات العربية، من أجل أن نكون قادرة على نطق تعبيرات مختلفة أو شيهها، لكي تكون قادرًا على التمييز بين جمل القراءة الطويلة والقصيرة، يمكنه التعبير عن رغبات قلبه باستخدام بحموعة من الرموز التي تتوافق مع قواعد النحوية، يمكن التعبير عما يتبادر إلى الذهن باستخدام القواعد الصحيحة في كتابة الجمل العربية، يمكن استخدام أجزاء من قواعد اللغة العبية في تعبيراتم مثل المذكر و المؤنث، يمكن استخدام التعبيرات اللغوية المناسبة للعمر والنضج 
والموقف، يمكن تتبع واستكشاف المخطوطات العربية والأدب، يمكن التعبير عن تعبيرات واضحة ومفهومة عن نفسه، القدرة على التفكير باللغة العربية والتعبير عنها بسرعة في أي موقف أو ظروف. ع. مبادئ تدريس مهارة الكلام

لكي يكون تعلم الكالام جيدًا لغير العرب، من الضروري الانتباه إلى ما يلي يجب أن المعلم له قدرة عالية على هذه المهارة، ابدأ بأصوات محاثلة بين لغتين (متعلمي اللغة والعربية)، يجب على الباحث و والمدرب الانتباه إلى المراحل في تعليم الكلام، مثل البدء بمفردة سهلة تتكون من جملة واحدة وجملتين وما إلى ذلك.

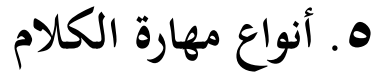

أ. أ. محادثة

محادثة هي وسيلة لتقديم لغة من خلال المحاورة، في تلك المحادثة يمكن أن تحدث بين المعلم و الطالب وبين الطلاب والطلاب، في حين أن إضافة إلى ومواصلة إثراء مفردات أكثر.

$$
\text { ب. تعبيرات شفهية }
$$

تعبيرات شفهية هو تمرين في إعداد مقالات شفهية تمدف إلى تطوير

$$
\text { قدرات الطلاب في التعبير عن أفكارهم ومشاعرهم. }
$$




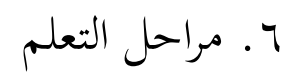

من بين المراحل العديدة لممارسة التحدث، وهي مساعد التدريب وتحديد الهوية، ممارسة أنماط الجملة (ممارسة النمط)، ممارسة التمرين، والقصة، والمناقشة، المناقشة والمقابالات والمسرحية والخطب. V . أساليب تعلم مهارة الكلام

فهم الأسلوب العربية من هذه التقنية هي تنفيذ خطط التعلم الفصل في شكل طرق وحيل مختلفة (خدعة) لتقديم مواد تعليمية من أجل تحقيق أهداف تعليمية

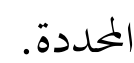

\section{و.نوع البحث}

البحث الذي أجراه الباحث هو البحث النوعي الوصفي باستخدام ثلاث تقنيات لجمع البيانات، وهي المقابالات والمالحظة والوثائق. ز. نتيـجة البحث

أن صعوبات التعلم مهارة الكالام ينبت من نفس المتكلم و خارجه كما في البيان الأتي البيان : أن علامة V تدل على أمن المشتركين يصعبون في تلك المرحلة و علامة X لا يصعبون في ذلك. 
أ. صعوبات في تعلم مهارة الكلام باللغة العربية على المثاركين في دورة اللغة

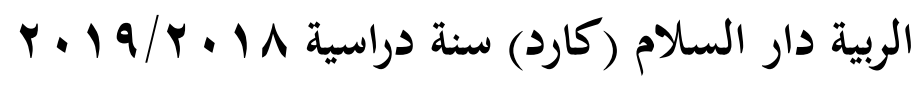

بناءً على الشرح الوارد أعلاه، هناك العديد من العوامل المثبطة للقدرة على التحدث باللغة العربية في دورات رارانج العربية لدروس السلام (كارد)، وهي العوامل

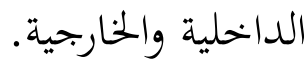

$$
\text { ( ) العوامل الداخلية }
$$

العوامل الداخلية هي العوامل التي تنشأ مباشرة من المشاركين أنفسهم. هذا العامل الداخلي هو الأكثر تأثيراً في نجاح شخص ما في تحقيق شيء ما.

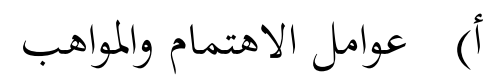

الاهتمام هو شعور شخص ما بأن ما تم تعلمه أو القيام به مفيد له. يعد منح المكافآت أو المكافآت أحد الأساليب التي تعتبر ناجحة في تطوير اهتمام المشاركين في الدورة. كما هو موضح، يتعلم الطفل بشدة ويستطيع الإجابة على جميع الأسئلة في اختبار أو اختبار، لذلك فإن المعلم سوف يكافئ الطفل بتقدير عالٍ وثناء وجوائز. بفضل هذه الجائزة، سيتعلم الطفل بمنتهى الجحية ويكون أكثر حماسة (شيف

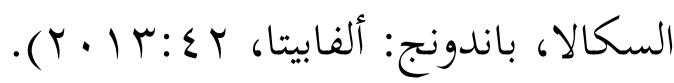


الغرض من الجائزة في التعلم هو أنه بمجرد أن يتلقى الشخص على جائزة كانت

تقوم به الجائزة بشكل جيد، وقال انه يجب أن تستمر في امتلاك أنشطة التعلم في خارج الفصول الدراسية بينما الموهبة هي عامل تأثير كبير على عملية التعلم واحدة والنتائج·

موهبة شخص تختلف عن بعضها البعض في مختلف النواحي، وبين الآخرين في المصالح، وعلى شخصيته، الحالة المادية والسلوك الاجتماعي. تقريبا لا أحد ينكر أن التعلم في بحال يتوافق مع الموهبة سيزيد من فرص النجاح. ب) عدم إتقان المفردات

المفردات أو المفردات هي عامل حاسم للغاية في مهارات الاتصال. بلدون

إتقان المفردات، سيكون من الصعب على المشاركين في الدورة التدريبية أن يتفاعلوا مع

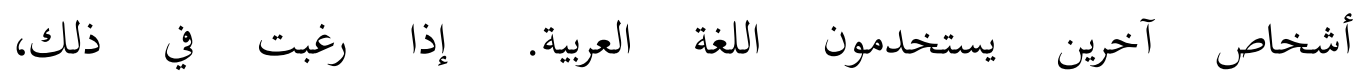
فمن السهللغاية بالنسبة للمشاركين في الدورة التدريبية أن يكونوا قادرين على إتقان المفردات، أي عن طريق إعطاء المعلم مهمة لكل مشارك في الدورة التدريبية ليحفظوا المفردات الجحديدة لكل اجتماع درس، محا سيساعد المشاركين في تطوير القدرة على التحدث باللغة العربية. إضافة إلى ذلك، فإن المفردات التي اعتمدتا الإندونيسية هي إضافة للاندونيسيين لتعلم اللغة العربية بسهوولة. في الواقع كلما زاد عدد الكلمات 
التي تأتي من الكلمات العربية والتي تم تحويلها بعد ذلك إلى المفردات الإندونيسية، كان من الأسهل تعزيز المفردات في ذاكرة الشخص. كما قال أحمد إزان في كتابه: "إن الاستفادة من المصطلحات الجحيدة والمفردات الجديدة يفيد بشكل كبير الأشخاص الذين يتعلمون اللغة العربية في إندونيسيا بدلاً من أمريكا والمملكة المتحدة ودول أخرى لأن الطلاب في إندونيسيا يكونون أسرع وأكثر بتمعًا للمفردات الجديدة". ج. أقل تفاعلا باستخدام اللغة العربية بعد أن تم حفظ العديد من عمليات الحفظ من قبل المشاركين في الدورة، حاول أن تتواصل قليلاً مع استخدام اللغة العربية مع زملائها في الفصل أو مع مدرسين حاليين. لأن اللغة هي درس يجب أن تكون طبيعته تدريبًا معتادًا ومستمرًا لإتقانه حقًا. يهدف التحدث إلى تمكين الطلاب من التواصل بشكل جيد ومنطق إلى حد ما مع اللغة التي يتعلموفا. من عملية الاتصال، يكتسب البشر الخبرة والتعلم. لأن اللغة الجحيدة التي يدرسوفا لا يمكن أن تكون آخر موضوع أو موضوع مدرسي كما هو، يجب توصيله.

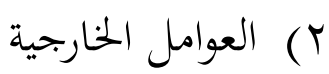
العوامل الخارجية هي العوامل التي تنشأ من خارج الدورة التدريبية المشاركين الذين يؤثر وجودهم أيضًا على معدل بنحاح المشاركين في الدورة في تحقيق شيء ما. 
فيما يلي بعض العوامل الخارجية التي تمنع القدرة على التحدث باللغة العربية لدى المشاركين في دورة جيل دار السلام العربية. أ. العوامل البيئية اللغوية

البيئة هي المكان الذي يقوم فيه المشاركون في الدورة بالقيام بأنشطة يومية. تظهر الحقائق أن العوامل البيئية هي مشكلة في تعلم اللغة العربية في إندونيسيا. من من المفترض أنه إذاكنت تريد أن تكون قادرًا على التحدث باللغة العبية، فيجب أن يكون هناك بيئة خاصة باللغة العربية حيث لا يُسمح للمشاركين في البيئة بالتواصل باستخدام لغات أخرى غير العربية. كما قال أجيف هرماوان في كتابه : "مع هذه البيئة، سيضطر كل طالب إلى استخدام اللغة، بحيث يكون تطوير إتقان اللغة التي يتعلمها أسرع نسبيًا مقارنة بأولئك الذين ليسوا في بيئة اللغة". إذا تم تنفيذ ذلك، فسيساعد المشاركين في تطويرهم على أن يكونوا قادرين على التحدث باللغة العربية بشكل صحيح، ولكن بالطبع يجب أن تكون هذه البيئة أيضًا تحت إشراف الوصاية. ب. حلفية المشاركين في الدورة ودوافع التعلم خلفية للمشاركين في الدورة، مثل الجاوية أو مادورا أو السندوية والمدارس الداخلية الإسلامية والمدارس الداخلية غير الإسلامية والأحفاد وغيرهم. في هذه الحالة، تسأل الباحث عن سبب شعور جميع المشاركين في دورة دار السلام تقريبًا بصعوبة في التحدث 
باللغة العربية نظرًا لخلفية المشاركين في الدورة ودوافعهم في التعلم، أي تسعة وعشرون من كل ثلاثين مشارگًا شعروا أن الصعوبة في مهارات التحدث. ولكن إذا نظرت عن كثب، فإن الصعوبات اللغوية مثل المفردات وترتيب الكلمات وقواعد اللغة تكون أكثر صعوبة وإلحاحًا من هذا. وبالتالي، فإن إجادة التعلم في دورات اللغة العربية ليس فقط صعوبة اللغة التي تشكل عائقًا أمام تعلم مهارات التحدث للمشاركين في الدورة، ولكن يجب أيضًا مراعاة الصعوبات غير اللغوية.

من الشرح أعلاه، من الواضح أن الصعوبات التعلم على التحدث باللغة العربية لدى المشاركين في دورة اللغة العربية دار السلام (كارد) الذي حصل عليه الباحثون مباشرة من الملاحظات يتضمن عوامل داخلية وخارجية والتي إذا لم يتم تصحيحها، فستظل مشاكل اللغة العربية في دورة دار السلام العربية (كارد). ب. خطوات لحل الصعوبات في تعلم مهارة الكالام باللغة العربية على المشاركين

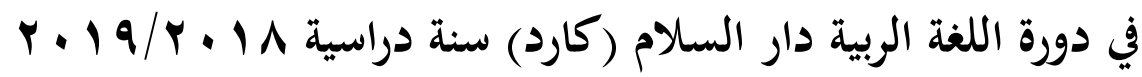
من أجل تحقيق هدف تعلم اللغة العربية جيدًا، يجب أن تكون العلاقة بين المشاركين في الدورة والمعلم متشابكة جيدًا أيضًا، لأنه مع وجود علاقة جيدة، سيؤدي ذلك بوضوح إلى تعاون قوي لتحقيق أهداف التعلم، مثل تقديم مدخلات للتغلب على المشكلات في القدرة تكلم بالعربية. 
يجب إكمال كل عقبة تواجه تعلم مهارة الكالام اللغة العربية على الفور حتى

يتم تحقيق أهداف التعلم المتوقعة، والتي تكون قادرة على التحدث باللغة العربية

$$
\text { بشكل صحيح وصحيح. لكن كل مشكلة لها حل في حلها. }
$$

في عملية التعلم، هناك العديد من الشروط التي يجب أن يمتلكها المشاركون

في الدورة من أجل الحصول على أقصى قدر من مخرجات التعلم، كما ذكر

ساجالا في كتابه.

لكي ينجح الطلاب، هناك حاجة إلى بعض المتطلبات، بما في ذلك:

() قدرة تفكير عالية للمشاركين في الدورة، ويتميز ذلك بالتفكير الناقد والمنطقي

والمنهجي والموضوعي.

Y) تسبب اهتماما كبيرا في الموضوعات.

ب) يمكن تطوير المواهب والاهتمامات الخاصة للمشاركين في الدورة وفقًا لقدراتم.

ع) إتقان المكونات الأساسية اللازمة لمواصلة التعلم في المدرسة.

0) إتقان لغة أجنبية واحدة .

7) (7) (الاستقرار النفسي.

( الصحة البدنية.

人) بيئة هادئة.

9) حياة اقتصادية كافية. 
• ( ) اتقان تقنيات التعلم في المدرسة وخارج المدرسة.

لا يواجه المشاركون في الدورة التدريبية كل مشكلة في تعلم مهرة الكالام اللغة العربية

فحسب، بل غالبًا ما تصبح أيضًا عائقًا أمام المعلمين. بما في ذلك:

( ) بجب أن يكون المعلمون قادرين على التأكيد على المشاركين في الدورة التدريبية

$$
\text { بكيث يكون أكثر في إتقان المفردات. }
$$

Y) فيما يتعلق ببيئة التعلم، كان المشاركون في الدورة أقل دعمًا، واقترحت المدرسة

أن يتكلم المشاركون دائمًا باللغة العربية طالما كانوا في البيئة المدرسة.

ب) فيما يتعلق بتخصيص وقت لتعلم اللغة العربية والذي يعتبر ضئياً للغاية، يمكن

للمدرسين العرب التفاعل مع الطلاب باستخدام دروس إضافية في الفصل

يمكن أن تضيف نظرة ثاقبة ومعرفة باللغة العربية إلى المشاركين في الدورة.

§) عقدت مدرسة درسا خاصا لممارسة التحدث أنه في حالة توفر مدرسين في

هذه العملية أمثلة كاملة من المحادثات التي رافقت مع معناها.

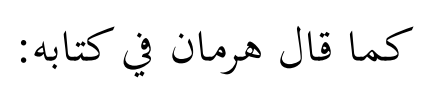

"هذه التقنية عبارة عن تمرين في تقليد وحفظ الحوارات المتعلقة بالحالات والفرص المختلفة. " من خلال هذا التمرين، من المتوقع أن يكون الطلاب قادرين على تحقيق إجادة جيدة في المحادثات التي يتم إجراؤها بطريقة عادلة وليست مصنوعة". 
ه) يجب أن يكون المعلمون قادرين على تحفيز المشاركين في الدورة ليكونوا سعداء

$$
\text { في تعلم اللغة العربية. }
$$

"تحفيز الطلاب مهم جدا للمعلمين. لتنفيذ هذه الأنشطة، يهتاج المعلم إلى فهم

المشاركين من الطلاب الجيدين، وهو قادر على توفير خبرات تعليمية مناسبة للطلاب".

عوامل من شأها أن تحفز المشاركين في الدورة على رغبة عالية في تعلم اللغة العربية

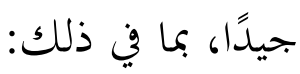

(1) بالإضافة إلى أغراض التواصل كما كذلك اللغات الأجنبية أمور أخرى،

تتعلق دراسة اللغة العربية أيضا إلى التعليم الديني و الطبيعي.

ب) جعلت اللغة العربية لغة دولية من قبل الأمم المتحدة.

ب) لغرض تعلم اللغة العربية ومن المتوقع أن يكون المشاركون في الدورة قادرين

على التواصل باللغة العربية جيدًا، فإن الخطوات المذكورة أعلاه جيدة جدًا

إذا كانت جميع عناصر التعليم والتدريس في دورة أجيال العربية دار السلام

(كارد) ترغب في الالتفات إليها. 
من نتائج البحث عن العوامل الصعوبات تعلم القدرة على التحدث

باللغة العربية لمشاركين في دورة اللغة دار السالام (كارد) سنة دراسية

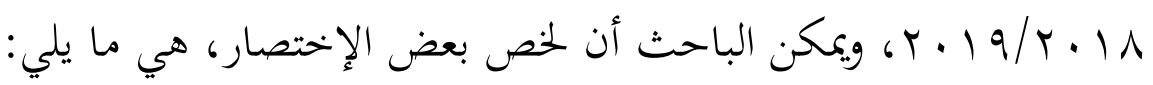

() إن القدرة على التحدث بالعربية للمشاركين في دورة اللغة دار السالام

(كارد) لا تزال لم يتوفر إلى حد الأقصى في تركييب الكلمات أو الجمل

العربية لأن هناك العديد من العقبات التي يواجهها المشاركون في الدورة

مثل عدم إتقان المفردات وقلة معرفتهم القواعد والتراكيب اللغوية.

ب) ينقسم عامل تثبيط القدرة على التحدث باللغة العربية في دورة اللغة دار

السلام إلى قسمين هما العوامل اللغوية والعوامل غير اللغوية. 
وتشمل العوامل اللغوية، عوامل الاهتمام والموهبة للمشاركين في الدورة التدريبية في تعلم اللغة العربية ، وذلك بسبب الافتقار إلى إتقان المفردات والعوامل التي تفتقر إلى التفاعل باستخدام اللغة العربية. والعوامل غير اللغوية هي، العوامل البيئية التي لا تستخدم اللغة العربية عند التواصل ، والعوامل في تعلم اللغة العربية غير موجودة.

r) خطوات لحل الصعوبات التي تعترض المشاركين في التحدث باللغة العببية. دورات اللغة العربية دار السلام (كارد) مع القائمين على العمل والمديرين القادرين على العمل بشكل جيد في الحل على المشاكل التي تعيق تطور المشاركين في دورة تعلم اللغة العربية، أي عن طريق كثرة حفظ المفردات على حد أكثر من العادة ضبطا تاما وكثرة التطبيق اللغوي كلاما وكشرة التمارين لتتمرين تركيب الجمل العربية، ثم إنشاء بيئة اللغة العربية التي يجب على كل مشارك الدورة استخدام اللغة العربية عندما تكون في البيئة. أخيرا أن التشجيع الطلاب هي خطوات لنمو كفائتهم في مهارة اللغوية فلذلك لابد أن نكثر المسابقات، المناقشات، الندوات ورحل العلمية إن 


\section{قائمة المراجع}

A.S Rahman, Kaserun. 2010. Kamus Modern Indonesia - Arab Al Kamal. Pustaka Progresif Surabaya.

Abd. Mutholib, Ahmad. 2003. Kesulitan-Kesulitan Belajar Bahasa Arab. Surabaya: Darul Fikri.

Abd. Wahab Dan Mamlu Atul Ni'mah. 2012. Memahami Konsep Dasar Pembelajaran Bahasa Arab. Malang: Uin-Maliki Press.

Acep Hermawan. 2011. Metodelogi Pembelajaran Bahasa Arab. Bandung: Rosdakarya.

Azhar Arsyad. 2003. Bahasa Arab Dan Metode Pengajarannya. Yogyakarta: Pustaka Pelajar.

Chaer, Abdul, 2004. Sosiolinguistik Perkenalan Awal. Jakarta: Rineka Cipta.

Djama'an Satori Dan Aan Komariah. 2013. Metodologi Penelitian Kualitataif. Bandung: Alfabeta.

Izzan Ahmad. 2011. Metodelogi Pembalajaran Bahasa Arab. Bandung: Humaniora.

Lexi J. Moleong. 2011. Metodologi Penelitian Kualitatif. Jakarta: Rineka Cipta.

Sugiono. 2014. Metodologi Pendidikan Kuantitatif, Kualitatif, Dan $D \& R$. Bandung: Alfabeta.

Suharsimi Arikunto. 2010. Prosedur Penelitian Suatu Pendekatan Praktik. Jakarta: Rineka Cipta.

Syalbi Ahmad, 1980. Pembelajaran Bahasa Arab Untuk Selain Arab. Perpustakaan Nahdhoh Misriyyah.

Ya'qub Hindi, Ahmad. 2015. Muhammad. Kesulitan Belajar Bahasa Arab Selain Penutur Bahasa Arab. Jakarta: Syabakah Alukah. 